\title{
A weighted voltage model predictive control method for a virtual synchronous generator with enhanced parameter robustness
}

\author{
Leilei Guo, Zhiye Xu, Nan Jin", Yanyan Li and Wei Wang
}

\begin{abstract}
To address the problem of insufficient system inertia and improve the power quality of grid-connected inverters, and to enhance the stability of the power system, a method to control a virtual synchronous generator (VSG) output voltage based on model predictive control (MPC) is proposed. Parameters of the inductors, capacitors and other components of the VSG can vary as the temperature and current changes. Consequently the VSG output voltage and power control accuracy using the conventional MPC method may be reduced. In this paper, to improve the parameter robustness of the MPC method, a new weighted predictive capacitor voltage control method is proposed. Through detailed theoretical analysis, the principle of the proposed method to reduce the influence of parameter errors on voltage tracking accuracy is analyzed. Finally, the effectiveness and feasibility of the proposed method are verified by experimental tests using the Typhoon control hardware-in-the-loop experimental platform.
\end{abstract}

Keywords: Virtual synchronous generator, Model predictive control, Optimal voltage vector, Parameter error compensation

\section{Introduction}

The development of clean, efficient and renewable energy is one of society's top priorities, while electricity is the main way to use energy and is an indispensable part of people's lives and national development [1]. Distributed generation (DG), as an effective supplement to centralized power generation, is being increasingly widely used in power systems [2-4].

In order to ensure the quality of power supply for users while reducing the adverse effects when DGs are connected to the grid, the concept of the microgrid has been proposed. In most microgrids, the parallel operation of distributed and large power grids is achieved through inverters. Thus, how to effectively control the inverters so that the microgrid can be better connected to the large power grid is critical $[5,6]$. There are many control

*Correspondence: jinnan@zzuli.edu.cn

College of Electric and Information Engineering, Zhengzhou University of Light Industry, Zhengzhou, China strategies for voltage control of microgrid inverters, including: (1) constant power (PQ), (2) constant voltage and constant frequency (V/f) [7], and (3) droop control [8]. However, these methods lack inertia provision and damping characteristics, and thus are not conducive to the stability of the system [9] and in severe cases, the system may even collapse. Therefore, these conditions need to be considered in the control process, resulting in a complicated control system.

By introducing the rotor mechanical equation and the stator electrical characteristic equation of the synchronous generator in the control, the virtual synchronous generator (VSG) can make the inverter exhibit the characteristics of the synchronous generator. The VSG provides inertia and damping, and helps maintain the stability of the power system. This makes the study the grid-connected inverter control method based on a VSG of significance $[10,11]$.

Much research has been conducted on the application of VSG control schemes. In [12], the method of using a 
VSG to improve the frequency stability of a photovoltaic microgrid is studied, where the VSG control emulates the inertial response and system damping by injecting power into the energy storage system. Reference [13] studies the VSG control of the grid-connected inverter in a microgrid. Using the VSG algorithm as the power outer loop, a voltage and current double closed-loop system is designed to improve stability. However, the above VSG controls are based on the PWM method, requiring the design of multiple proportional integral (PI) controllers which are difficult to design and tune.

In recent years, there has been increased interest in the application of model predictive control (MPC) technology in the field of power electronics [14-17], as it does not require the design of a PI controller and a PWM algorithm. In [16], an adaptive compensation algorithm based on inherent frequency is proposed to estimate the power grid voltage, and an MPC strategy is then designed using the estimated grid voltage to reduce current ripple. In [17], an improved MPC-based common-mode voltage $(\mathrm{CMV})$ reduction method is proposed to reduce the CMV and eliminate the CMV spikes for two-level voltage source inverters. However, there has been limited research on the control method combining VSG and MPC. In [18], an improved finite-set MPC is proposed for the inner loop. This has a simple control structure, faster dynamic response, and enhanced control bandwidth and stability. In the outer control loop, a simplified VSG without a phase-locked loop is employed to realize active power sharing and inertia emulation. Nevertheless, the above MPC methods often assume that the inverter model used for prediction is consistent with the actual system. The parameter errors of the inverters, errors which affect system control performance, are not considered [19].

In [20], a new adaptive control scheme that uses the average switching frequency to adjust the parameter values of the predictive model online is presented. This control scheme can keep the average switching frequency within an acceptable operational margin, while improving the stability of the control system. In [21], the extended control vectors are adopted to improve the control precision, and an indirect reference vector strategy is developed to avoid the penalty of heavy computation time and increase robustness. In [22], a robust MPC method is proposed based on the current error. By studying the effects of parameter changes on the current error, two PI controllers are then adopted to extract accurate inductance and flux linkage parameters. However, the error compensations in [21, 22] require several PI controllers, which as we have said are difficult to design and tune. In addition there is a lack of detailed theoretical support.
In this paper, to achieve the control of VSG without using a PI controller and a PWM algorithm, and to enhance parameter robustness, a new weighted voltage MPC method for the VSG is proposed. The main improvements of this work over existing studies are:

(1) Detailed theoretical analysis of the influence of parameter changes on the conventional MPC for the VSG is conducted to show its sensitivity to parameter errors.

(2) A new weighted voltage MPC method is proposed for the VSG, which uses the weighted sum of the sampled voltage and the predicted one to predict the voltage at the next period.

(3) A detailed theoretical analysis is carried out to show the influence of parameter changes on the proposed weighted voltage MPC method. This analysis verifies the enhanced robustness of the proposed method against parameter variations.

(4) The outer-loop of the VSG, which generates the reference voltage for the proposed weighted voltage MPC method, is designed to control the active and reactive power, and to solve the problem of insufficient system inertia.

(5) Compared to the conventional VSG control method, the proposed method can achieve direct output voltage predictive control without a PI controller and a PWM algorithm.

(6) A PE-Expert4 and Typhoon-602 + based hardwarein-the-loop experimental study is carried out, which further verifies the feasibility and effectiveness of the proposed strategy.

\section{VSG model and its control}

The VSG enables grid-connected inverters to emulate the operating mechanism of synchronous generators, so they have the same operational mechanism and external characteristics as traditional synchronous generators. Such chararacteristics are: torque inertia, active power frequency modulation, and reactive power voltage regulation.

The topology of the inverter with an output LC filter is shown in Fig. 1, where the DC power supply and the three-phase inverter with the output LC filter can be equivalent to the VSG. The control block diagram of the VSG is shown in Fig. 2, which mainly includes the VSG model and its core control algorithm. The former emulates the electromagnetic relationship and mechanical movement of the synchronous generator, while the latter mainly adopts frequency and voltage regulation to realize active and reactive power control. 


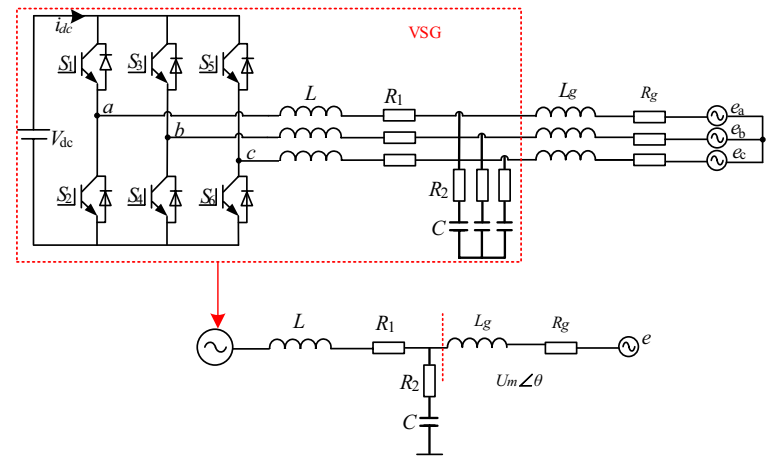

Fig. 1 Topological map of the inverter with output LC filter

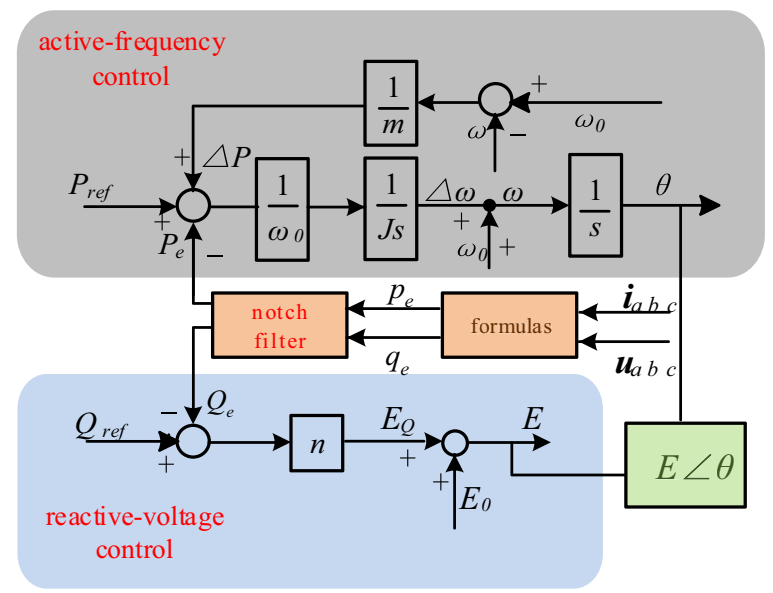

Fig. 2 Control system block diagram of the VSG

\subsection{Active power frequency control}

The active power frequency control of a VSG is by emulating the rotor equation of the synchronous generator. The second-order equation of the traditional synchronous generator is used for modeling [23]:

$$
\left\{\begin{array}{l}
J \omega_{0} \frac{d \omega}{d t}=P_{\mathrm{m}}-P_{\mathrm{e}}-D_{\mathrm{p}}\left(\omega-\omega_{0}\right) \\
\frac{d \theta}{d t}=\omega
\end{array}\right.
$$

where $P_{\mathrm{m}}$ and $P_{\mathrm{e}}$ are the mechanical and average power of the synchronous generator, respectively. $J$ is the rotary inertia of the synchronous generator. $D_{\mathrm{p}}$ is the damping coefficient. $\omega$ and $\omega_{0}$ are the angular frequencies of the synchronous generator and grid, respectively. $\theta$ is the rotation angle of the generator rotor relative to the a-phase stator winding. The virtual mechanical power of the VSG is calculated by:

$$
P_{\mathrm{m}}=P_{\text {ref }}+\Delta P=P_{\text {ref }}+\frac{1}{\mathrm{~m}}\left(\omega_{0}-\omega\right)
$$

where $P_{\text {ref }}$ is the reference active power. $\Delta P$ is the output of the VSG frequency regulator. $m$ is an active power adjustment factor which contains the damping coefficient $D_{\mathrm{p}}$. The instantaneous electromagnetic power $p_{e}$ of the VSG is calculated by:

$$
p_{e}=1.5\left(u_{c \alpha} i_{\alpha}+u_{c \beta} i_{\beta}\right)
$$

where $i_{\alpha}, i_{\beta}$ are the grid currents in the $\alpha \beta$ coordinate, and $u_{c \alpha}, u_{c \beta}$ are the voltages at the filter bus in the $\alpha \beta$ coordinate. The output average active power of the VSG $P_{\mathrm{e}}$ can be obtained through a notch filter to reduce power fluctuation [12], as:

$$
P_{\mathrm{e}}=\frac{s^{2}+\omega_{\mathrm{m}}^{2}}{s^{2}+2 \delta \omega_{\mathrm{m}} s+\omega_{\mathrm{m}}^{2}} p_{\mathrm{e}}
$$

where $\omega_{\mathrm{m}}$ is the harmonic angular frequency of the notch filter and $\delta$ is the quality factor.

It can be seen from the above formulas that $J$ emulates the moment of inertia. This can reduce system frequency fluctuation after disturbances. The frequency returns to the rated value by adjusting $P_{\mathrm{m}}$ and $P_{\mathrm{e}}$. The difference between the VSG output angular frequency $\omega$ and the grid frequency $\omega_{0}$ is used as feedback for the active power-frequency control to obtain the VSG output angular frequency $\omega$, while the phase reference $\theta$ is obtained by integration of $\omega$.

\subsection{Reactive power voltage control}

For the VSG reactive power voltage control, the control block diagram is shown in Fig. 2, where $E$ is the reference voltage amplitude and consists of two parts:

$$
E=E_{0}+E_{\mathrm{Q}}=E_{0}+n\left(Q_{\mathrm{ref}}-Q_{\mathrm{e}}\right)
$$

where $E_{0}$ is the no-load EMF of the VSG. $E_{\mathrm{Q}}$ is the output voltage of the reactive power regulator. $n$ is the reactive power adjustment factor. $Q_{\text {ref }}$ is the reference reactive power, and $Q_{\mathrm{e}}$ is the output average reactive power of the VSG, obtained as:

$$
Q_{\mathrm{e}}=\frac{s^{2}+\omega_{\mathrm{m}}^{2}}{s^{2}+2 \delta \omega_{\mathrm{m}} s+\omega_{\mathrm{m}}^{2}} 1.5\left(u_{\mathrm{c} \beta} i_{\alpha}-u_{\mathrm{c} \alpha} i_{\beta}\right)
$$

The reference voltage amplitude $E$ and the angle $\theta$ obtained by the active power frequency control are combined to derive the reference voltage for the inner-loop voltage predictive control.

\section{Voltage model predictive control for VSG 3.1 Principle of voltage model predictive control}

The 2-level 3-phase inverter shown in Fig. 1 has 8 voltage vectors, as shown in Fig. 3. From Fig. 1, the dynamic vector equation can be expressed as: 


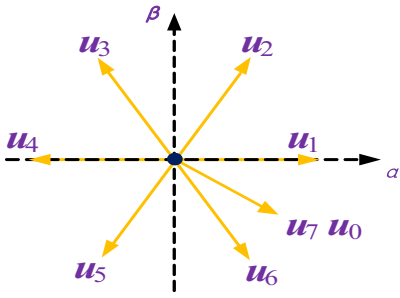

Fig. 3 The output voltage vectors

$$
\left\{\begin{array}{l}
L \frac{d \boldsymbol{i}_{\mathrm{f} \alpha \beta}}{d t}=\boldsymbol{u}-\boldsymbol{u}_{\mathrm{c} \alpha \beta}-\boldsymbol{i}_{\mathrm{f} \alpha \beta} R_{1} \\
C \frac{d\left[\boldsymbol{u}_{c \alpha \beta}-R_{2}\left(i_{\mathrm{f} \alpha \beta}-i_{\alpha \beta}\right)\right]}{d t}=i_{\mathrm{f} \alpha \beta}-i_{\alpha \beta}
\end{array}\right.
$$

where $L$ is the output filter inductance of the inverter, and $C$ is the filter capacitor. $R_{1}$ and $R_{2}$ are the parasitic resistances of the filter inductor and filter capacitor, respectively. $\boldsymbol{i}_{\mathrm{f} \alpha \beta}$ and $\boldsymbol{i}_{\alpha \beta}$ are the current vectors flowing through the filter inductor $L$ and the grid-side inductor $L_{g}$ in the $\alpha \beta$ coordinate, respectively. $\boldsymbol{u}$ is the output voltage vector of the inverter, and $\boldsymbol{u}_{c \alpha \beta}$ is the voltage vector at the filter bus. Equation (7) can be discretized as:

$\left\{\begin{aligned} & \boldsymbol{i}_{\mathrm{f} \alpha \beta}(k+1)=\frac{T_{s}}{L}[\left.\boldsymbol{u}_{\alpha \beta}(k)-\boldsymbol{u}_{\mathrm{c} \alpha \beta}(k)-\boldsymbol{i}_{\mathrm{f} \alpha \beta} \cdot R_{1}\right]+\boldsymbol{i}_{\mathrm{f} \alpha \beta}(k) \\ & \boldsymbol{u}_{\mathrm{c} \alpha \beta}(k+1)=\frac{T_{s}}{C}\left[\boldsymbol{i}_{\mathrm{f} \alpha \beta}(k+1)-\boldsymbol{i}_{\alpha \beta}(k)\right]+\boldsymbol{u}_{\mathrm{c} \alpha \beta}(k) \\ &+R_{2}\left[\boldsymbol{i}_{\mathrm{f} \alpha \beta}(k+1)-\boldsymbol{i}_{\alpha \beta}(k+1)-\boldsymbol{i}_{\mathrm{f} \alpha \beta}(k)+\boldsymbol{i}_{\alpha \beta}(k)\right]\end{aligned}\right.$

where $T_{\mathrm{s}}$ is the sampling period.

Assuming that $\boldsymbol{i}_{\alpha \beta}(k)=\boldsymbol{i}_{\alpha \beta}(k+1)$, the prediction functions of $\boldsymbol{i}_{\mathrm{f} \alpha \beta}$ and $\boldsymbol{u}_{c \alpha \beta}$ can be obtained as:

$$
\left\{\begin{array}{l}
\boldsymbol{i}_{\mathrm{f} \alpha \beta}(k+1)=\frac{T_{\mathrm{s}}}{L}\left[\boldsymbol{u}_{\alpha \beta}(k)-\boldsymbol{u}_{\mathrm{c} \alpha \beta}(k)\right]-\frac{T_{s}}{L} \boldsymbol{i}_{\mathrm{f} \alpha \beta}(k) \cdot R_{1} \\
\quad+\boldsymbol{i}_{\mathrm{f} \alpha \beta}(k) \\
\boldsymbol{u}_{\mathrm{c} \alpha \beta}(k+1)=\frac{T_{\mathrm{s}}}{C}\left[\boldsymbol{i}_{\mathrm{f} \alpha \beta}(k+1)-\boldsymbol{i}_{\alpha \beta}(k)\right]+\boldsymbol{u}_{\mathrm{c} \alpha \beta}(k) \\
\quad+R_{2}\left[\boldsymbol{i}_{\mathrm{f} \alpha \beta}(k+1)-\boldsymbol{i}_{\mathrm{f} \alpha \beta}(k)\right]
\end{array}\right.
$$

From (9), once the current $\boldsymbol{i}_{\mathrm{f} \alpha \beta}(k)$ and the capacitor voltage $\boldsymbol{u}_{\mathrm{c} \alpha \beta}(k)$ at the current time $t_{k}$ are sampled, the inductor current $\boldsymbol{i}_{\mathrm{f} \alpha \beta}(k+1)$ at time $t_{k+1}$ can be predicted. Then, by sampling the grid current $\boldsymbol{i}_{\alpha \beta}(k)$, the voltage $\boldsymbol{u}_{\mathrm{c} \alpha \beta}(k+1)$ at time $t_{k+1}$ can be further predicted. The cost function is constructed by sampling the reference voltage generated by the virtual synchronous control as:

$$
g=\left|u_{\alpha}^{*}-u_{\mathrm{c} \alpha}(k+1)\right|+\left|u_{\beta}^{*}-u_{\mathrm{c} \beta}(k+1)\right|
$$

where $u_{\alpha}^{*}$ and $u_{\beta}^{*}$ are the reference voltages, whereas $u_{\mathrm{c} \alpha}$ $(k+1)$ and $u_{\mathrm{c} \beta}(k+1)$ are the predicted voltage values at the next sampling instant.

The voltage vectors corresponding to the eight switching states are sequentially substituted into (10). By comparing the cost functions, the optimal switch state corresponding to the voltage vector that minimizes the

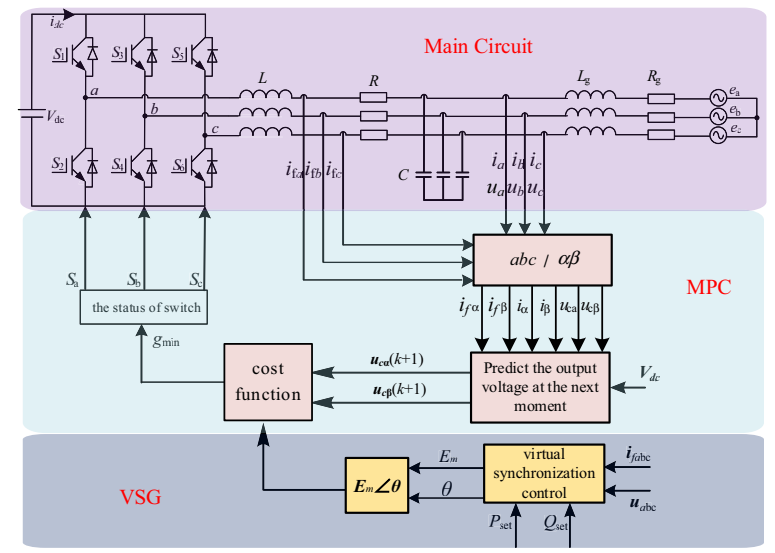

Fig. 4 VSG control block diagram based on voltage model predictive control

cost function is selected and applied in the next control period. The VSG control block diagram based on voltage predictive control is shown in Fig. 4.

\subsection{Influence of parameter error on control performance} As the MPC strategy is a model-based control method, parameter changes can influence its control performance. Considering the temperature and current change during inverter operation, the actual values of the device parameters (filter inductance, filter capacitance, resistance, etc.) often deviate from their theoretical values. This deviation may affect the entire process of voltage predictive control. In the following, the effects of the parameter errors on the voltage predictive control are analyzed.

Assuming that the actual inductance is $L$ and the modeled one is $L_{0}$, the prediction function in (9) can be rewritten as (11) using the modeled inductance $L_{0}$.

$$
\left\{\begin{array}{l}
\boldsymbol{i}_{\mathrm{f} \alpha \beta 0}(k+1)=\frac{T_{\mathrm{s}}}{L_{0}}\left[\boldsymbol{u}_{\alpha \beta}(k)-\boldsymbol{u}_{\mathrm{c} \alpha \beta}(k)\right]-\frac{T_{\mathrm{s}}}{L_{0}} \boldsymbol{i}_{\mathrm{f} \alpha \beta}(k) \cdot R_{1} \\
\quad+\boldsymbol{i}_{\mathrm{f} \alpha \beta}(k) \\
\boldsymbol{u}_{\mathrm{c} \alpha \beta 0}(k+1)=\frac{T_{\mathrm{s}}}{C}\left[\boldsymbol{i}_{\mathrm{f} \alpha \beta 0}(k+1)-\boldsymbol{i}_{\alpha \beta}(k)\right]+\boldsymbol{u}_{\mathrm{c} \alpha \beta}(k) \\
\quad+R_{2}\left[\boldsymbol{i}_{\mathrm{f} \alpha \beta 0}(k+1)-\boldsymbol{i}_{\mathrm{f} \alpha \beta}(k)\right]
\end{array}\right.
$$

Prediction errors, which are the difference between the predicted and actual values deduced based on (9) and (11), are depicted in (12).

$$
\left\{\begin{array}{l}
\Delta \boldsymbol{i}=\frac{T_{\mathrm{s}}\left(L-L_{0}\right)}{L_{0} L}\left[\boldsymbol{u}_{\alpha \beta}(k)-\boldsymbol{u}_{\mathrm{c} \alpha \beta}(k)-\boldsymbol{i}_{\mathrm{f} \alpha \beta}(k) \cdot R_{1}\right] \\
\Delta \boldsymbol{U}=\frac{T_{\mathrm{s}}}{C}\left(\boldsymbol{i}_{\mathrm{f} \alpha \beta 0}(k+1)-\boldsymbol{i}_{\mathrm{f} \alpha \beta}(k+1)\right) \\
\quad+R_{2}\left(\boldsymbol{i}_{\mathrm{f} \alpha \beta 0}(k+1)-\boldsymbol{i}_{\mathrm{f} \alpha \beta}(k+1)\right) \\
=\left(\frac{T_{\mathrm{s}}}{C}+R_{2}\right) \Delta \boldsymbol{i}
\end{array}\right.
$$

In the same way, assuming that the actual capacitance is $C$ while the modeled is $C_{0}$, the influence of the 
capacitance error on voltage predictive control can also be analyzed as:

$$
\Delta U=\frac{T_{\mathrm{s}}(C-C 0)}{C C 0}\left[\boldsymbol{i}_{\mathrm{f} \alpha \beta}(k+1)-\boldsymbol{i}_{\alpha \beta}(k)\right]
$$

The analysis of (12) and (13) shows that when the parameter values in the prediction model equal the actual ones in the circuit, i.e., $L=L_{0}$ and $C=C_{0}$, the current and voltage prediction errors are zero. However, if the parameter values in the prediction model are inconsistent with the actual ones, there will be a voltage prediction error and the optimal switch function selected according to the traditional MPC algorithm will lose its optimality. Consequently, it cannot achieve error-free tracking of the reference values and affects the control performance of the system.

\section{Voltage model predictive control with error compensation}

\subsection{Design of VMPC with error compensation}

From the above analysis, it can be seen that when the parameters in the prediction model do not match the actual values, system control performance will deteriorate. In order to reduce the influence of the parameter errors on control performance and improve the voltage control accuracy, a new weighted predictive voltage control method is proposed in this paper. The proposed method uses the weighted sum of the sampled voltage and the predicted one to predict the voltage at the next period, as shown in Fig. 5.

The prediction in (9) can be rewritten using the weighted sum of the sampled voltage and the predicted one considering the weighting factor $M$, to predict the voltage at the next period, as:

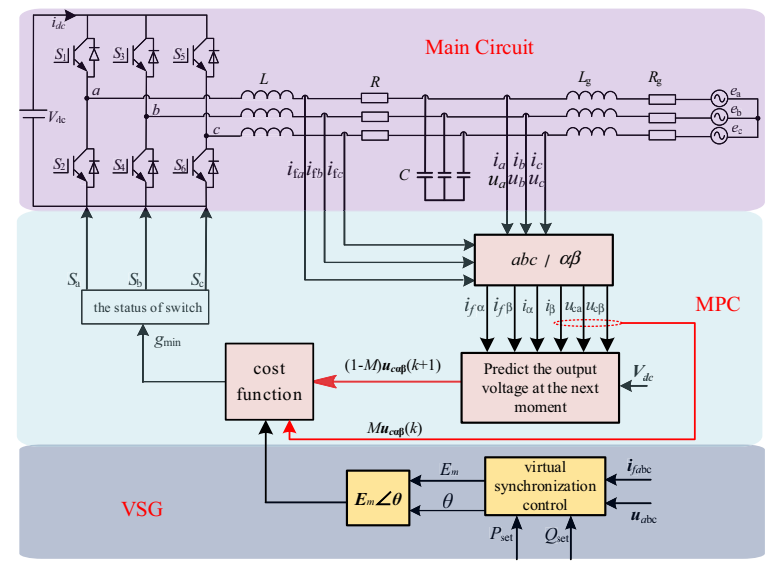

Fig. 5 VSG control block diagram based on the proposed weighted predictive voltage control method

$$
\left\{\begin{array}{l}
\boldsymbol{i}_{\mathrm{f} \alpha \beta}(k+1)=\frac{T_{\mathrm{s}}}{L}\left[\boldsymbol{u}_{\alpha \beta}(k)-\boldsymbol{u}_{\mathrm{c} \alpha \beta}(k)\right]-\frac{T_{\mathrm{s}}}{L} \boldsymbol{i}_{\mathrm{f} \alpha \beta}(k) \cdot R_{1} \\
\quad+\boldsymbol{i}_{\mathrm{f} \alpha \beta}(k) \\
\boldsymbol{u}_{\mathrm{c} \alpha \beta}(k+1)=(1-M) \boldsymbol{u}_{\mathrm{c} \alpha \beta}(k+1)+M \boldsymbol{u}_{\mathrm{c} \alpha \beta}(k) \\
=(1-M) \frac{T_{\mathrm{s}}\left[\boldsymbol{i}_{\mathrm{f} \alpha \beta}(k+1)-\boldsymbol{i}_{\alpha \beta}(k)\right]}{\quad} \quad+(1-M) R_{2}\left[\boldsymbol{i}_{\mathrm{f} \alpha \beta}(k+1)-\boldsymbol{i}_{\mathrm{f} \alpha \beta}(k)\right]+\boldsymbol{u}_{\mathrm{c} \alpha \beta}(k)
\end{array}\right.
$$

\subsection{Effectiveness analysis of the proposed weighted VMPC method}

The effectiveness of the proposed weighted voltage predictive control method in reducing the influence of parameter changes on the control error is analyzed in this subsection. When the inductance error exists, Eq. (11) can be rewritten as (15) based on (14), while (16) can be obtained from (15) and (9).

$$
\left\{\begin{array}{l}
\boldsymbol{i}_{\mathrm{f} \alpha \beta 0}(k+1)=\frac{T_{\mathrm{s}}}{L_{0}}\left[\boldsymbol{u}_{\alpha \beta}(k)-\boldsymbol{u}_{\mathrm{c} \alpha \beta}(k)\right]-\frac{T_{\mathrm{s}}}{L_{0}} \boldsymbol{i}_{\mathrm{f} \alpha \beta}(k) \cdot R_{1} \\
\quad+\boldsymbol{i}_{\mathrm{f} \alpha \beta}(k) \\
\boldsymbol{u}_{\mathrm{c} \alpha \beta 0}(k+1)=(1-M) \boldsymbol{u}_{\mathrm{c} \alpha \beta}(k+1)+M \boldsymbol{u}_{\mathrm{c} \alpha \beta}(k) \\
=(1-M) \frac{T_{\mathrm{s}}}{C}\left[\boldsymbol{i}_{\mathrm{f} \alpha \beta 0}(k+1)-\boldsymbol{i}_{\alpha \beta}(k)\right] \\
\quad+(1-M) R_{2}\left[\boldsymbol{i}_{\mathrm{f} \alpha \beta 0}(k+1)-\boldsymbol{i}_{\mathrm{f} \alpha \beta}(k)\right]+\boldsymbol{u}_{\mathrm{c} \alpha \beta}(k)
\end{array}\right.
$$

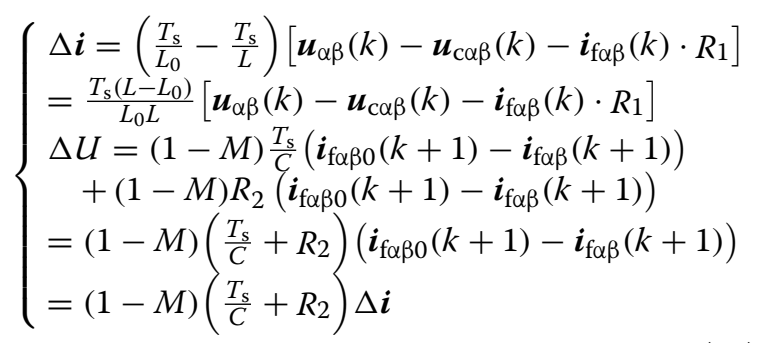

Comparing (16) with (12), if $M=1$, there is no voltage prediction, while if $|1-M|>1$, the impact of the inductance error will be further enlarged. However, with $|1-M|<1$, the voltage prediction error caused by the inductance error can be reduced. Therefore, choosing an appropriate value of $M$ can weaken the influence of the inductance error on voltage tracking and improve voltage control precision.

Similarly, when a capacitance error exists, there are:

$$
\begin{aligned}
& \left\{\begin{array}{c}
\boldsymbol{i}_{\mathrm{f} \alpha \beta}(k+1)=\frac{T_{\mathrm{s}}}{L}\left[\boldsymbol{u}_{\alpha \beta}(k)-\boldsymbol{u}_{\mathrm{c} \alpha \beta}(k)\right] \\
\quad-\frac{T_{\mathrm{s}}}{L} \boldsymbol{i}_{\mathrm{f} \alpha \beta}(k) \cdot R_{1}+\boldsymbol{i}_{\mathrm{f} \alpha \beta}(k) \\
\boldsymbol{u}_{\mathrm{c} \alpha \beta 0}(k+1)=(1-M) \boldsymbol{u}_{\mathrm{c} \alpha \beta}(k+1)+M \boldsymbol{u}_{\mathrm{c} \alpha \beta}(k) \\
=(1-M) \frac{T_{\mathrm{s}}}{C_{0}}\left[\boldsymbol{i}_{\mathrm{f} \alpha \beta}(k+1)-\boldsymbol{i}_{\alpha \beta}(k)\right] \\
+(1-M) R_{2}\left[\boldsymbol{i}_{\mathrm{f} \alpha \beta}(k+1)-\boldsymbol{i}_{\mathrm{f} \alpha \beta}(k)\right] \\
+\boldsymbol{u}_{\mathrm{c} \alpha \beta}(k)
\end{array}\right. \\
& \Delta U=(1-M) \frac{T_{\mathrm{s}}(C-C 0)}{C C 0}\left[\boldsymbol{i}_{\mathrm{f} \alpha \beta}(k+1)-\boldsymbol{i}_{\alpha \beta}(k)\right]
\end{aligned}
$$


Comparing (18) with (13), the same conclusion can be obtained, which means that choosing an $M$ that meets $|1-M|<1$ can enhance the parameter robustness of the proposed MPC strategy for VSG.

\section{Experimental results}

To verify the feasibility of the proposed VSG voltage MPC with error compensation, a hardware-in-the-loop experimental platform based on a Typhoon real-time simulator and PE-Expert4 as shown in Fig. 6 is established and studies are conducted. In the experiments, the main circuit of the grid-connected inverter is run in the Typhoon real-time simulator, and the VSG-MPC control algorithm is implemented in the PE-Expert4 (Table 1).

\subsection{Comparison with accurate parameters}

In order to verify the feasibility of the power outer loop control method with the compensation algorithm, the performance is compared with the traditional control methods without compensation. No parameter mismatch is considered here.

Figure 7 shows the results for the traditional power outer loop without compensation. The active power $P_{\text {ref }}$ changes from 500 to $2000 \mathrm{~W}$ in Fig. 7a. As can be seen, the actual output active power of the inverter follows the reference change while the reactive power has a small disturbance at the corresponding moment due to the sudden change of the active power. Similar phenomena can be observed during the reactive power $Q_{\text {ref }}$ change from 500 to 0 Var in Fig. 7b. In both cases, the actual active and reactive power outputs by the inverter are stabilized at the given values after the transients.

With the same active and reactive power changes, Fig. 8a, b show the experimental results of the power outer loop with the proposed compensation. Comparing Figs. 7 and 8, the results are almost the same, which means that the proposed weighted voltage predictive control method has the same control performance as the conventional method when the parameters are accurate.

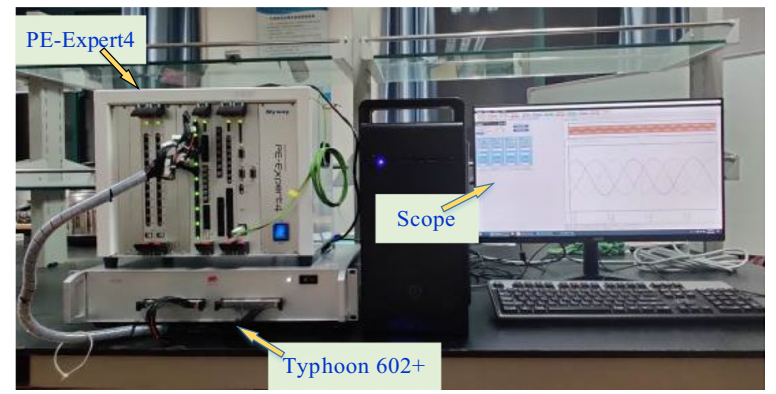

Fig. 6 Typhoon and PE-Expert4 hardware-in-loop experimental platform
Table 1 Simulation parameters

\begin{tabular}{ll}
\hline Parameters & Value \\
\hline DC voltage $V_{\text {dc }} N$ & 500 \\
Phase voltage of grid $e / N$ & 190 \\
Filter inductance $L / \mathrm{mH}$ & 6.4 \\
Resistance $R_{1} / \Omega$ & 0.1 \\
Filter capacitor $C / \mathrm{mF}$ & 0.6 \\
Resistance $R_{2} / \Omega$ & 0.1 \\
Grid-side inductance $\mathrm{Lg} / \mathrm{mH}$ & 1 \\
Grid-side resistance $R \mathrm{~g} / \Omega$ & 1.5 \\
Switching frequency $\mathrm{f}_{\mathrm{s}} / \mathrm{kHz}$ & 40 \\
Grid frequency $\mathrm{f} / \mathrm{Hz}$ & 50 \\
Droop coefficient $D_{\mathrm{q}}$ & 0.05 \\
Moment of Inertia $/ / \mathrm{kg} \mathrm{m}^{2}$ & 0.0000005 \\
Damping coefficient $D_{\mathrm{p}}$ & 0.0003 \\
Weight factor $M$ & 0.7 \\
\hline
\end{tabular}

Figures 9 and 10 further show the results during a sudden change of active power without and with error compensation, respectively. Figures $9 \mathrm{a}$ and 10a show the complete change process of the active power, while Figs. $9 \mathrm{~b}, \mathrm{c}$ and $10 \mathrm{~b}, \mathrm{c}$ show the details of the $\beta$-axis voltage changes in different time ranges during the transients. As can be seen, for both cases, when the active power changes suddenly from 500 to $2000 \mathrm{~W}$, the $\beta$-axis voltage values are increased. Comparing Figs. 9 and 10, it can be seen that the waveforms corresponding to the two methods are almost identical. Both methods produce satisfactory voltage waveforms and the differences between the actual and the reference $\beta$-axis voltages for the two

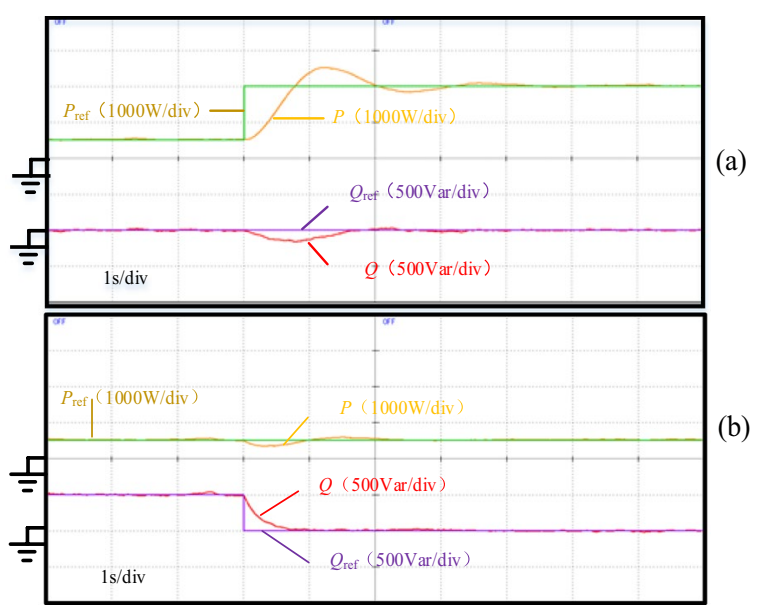

Fig. 7 Experimental waveforms of the power outer loop without compensation. a Active power $P_{\text {ref }}$ changes from 500 to 2000 W. b Reactive power $Q_{\text {ref }}$ changes from 500 to 0 Var 


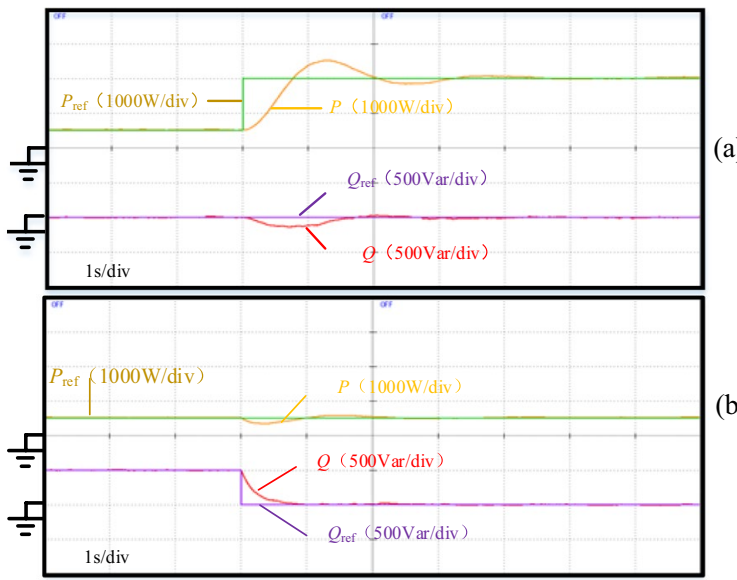

Fig. 8 Experimental waveforms of the power outer loop with compensation. (a) Active power $P_{\text {ref }}$ changes from 500 to 2000 W. (b) Reactive power $Q_{\text {ref }}$ changes from 500 to OVar

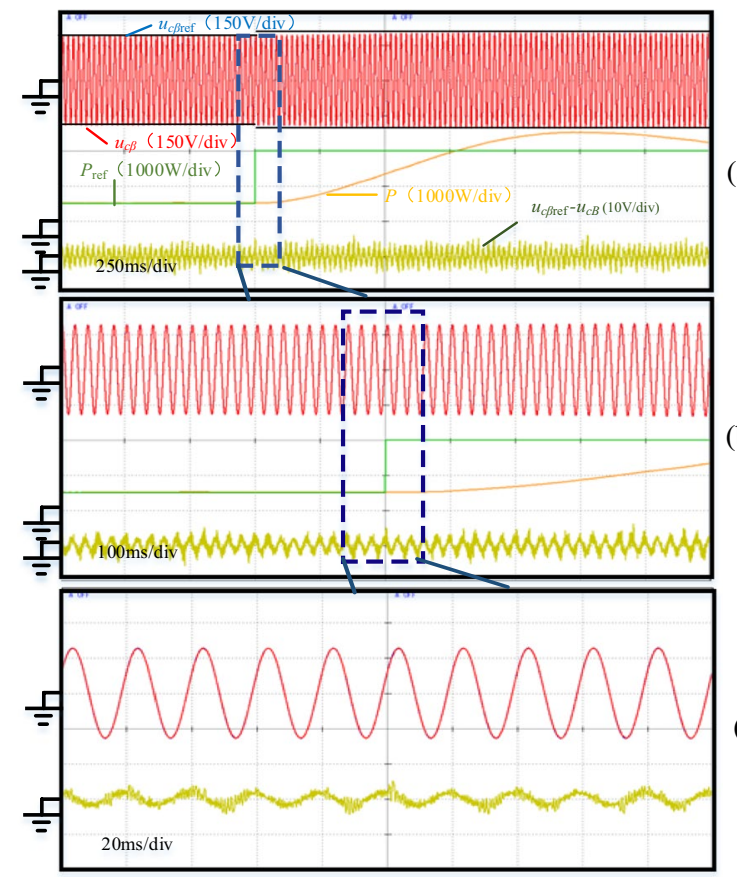

Fig. 9 Experimental waveforms during sudden change of active power without error compensation. a $250 \mathrm{~ms} / \mathrm{div}$. b $100 \mathrm{~ms} / \mathrm{div}$. c $20 \mathrm{~ms} / \mathrm{div}$

methods are almost identical in both dynamic and steady states.

Similarly, further experimental waveforms during reactive power change for the two methods are compared in Figs. 11 and 12. As seen there, when the reactive power changes suddenly from 500 to 0 Var, the $\beta$-axis voltage values are reduced, while the waveforms corresponding to the two methods are almost identical.

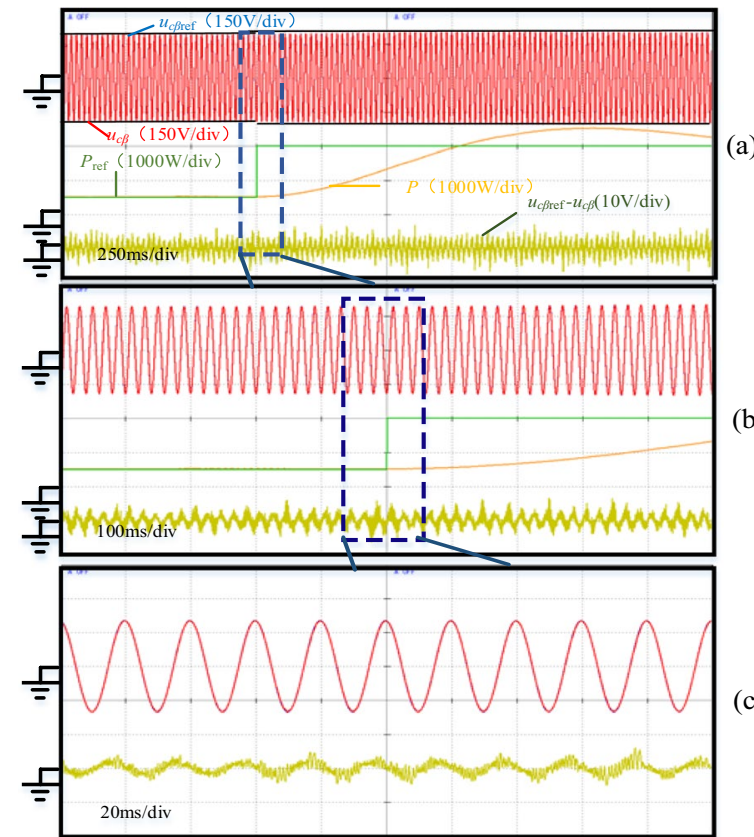

Fig. 10 Experimental waveforms during sudden change of active power with error compensation. a $250 \mathrm{~ms} / \mathrm{div}$. b $100 \mathrm{~ms} / \mathrm{div}$. c $20 \mathrm{~ms} /$ div

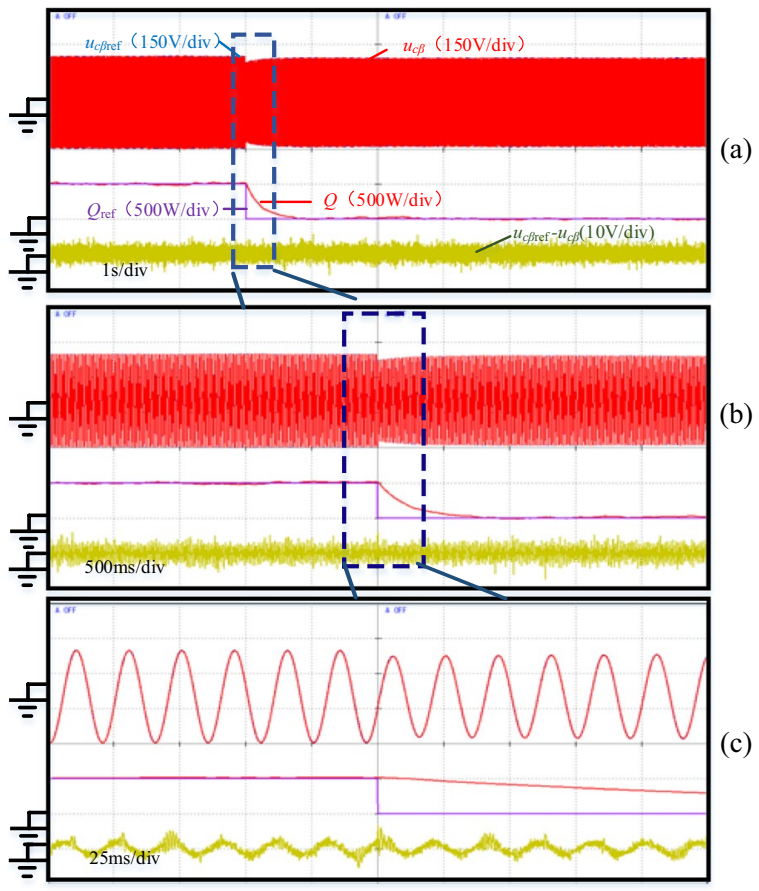

Fig. 11 Experimental waveforms during sudden change of reactive power without error compensation. a 1 s/div. b 500 ms/div. c 25 ms/ div 


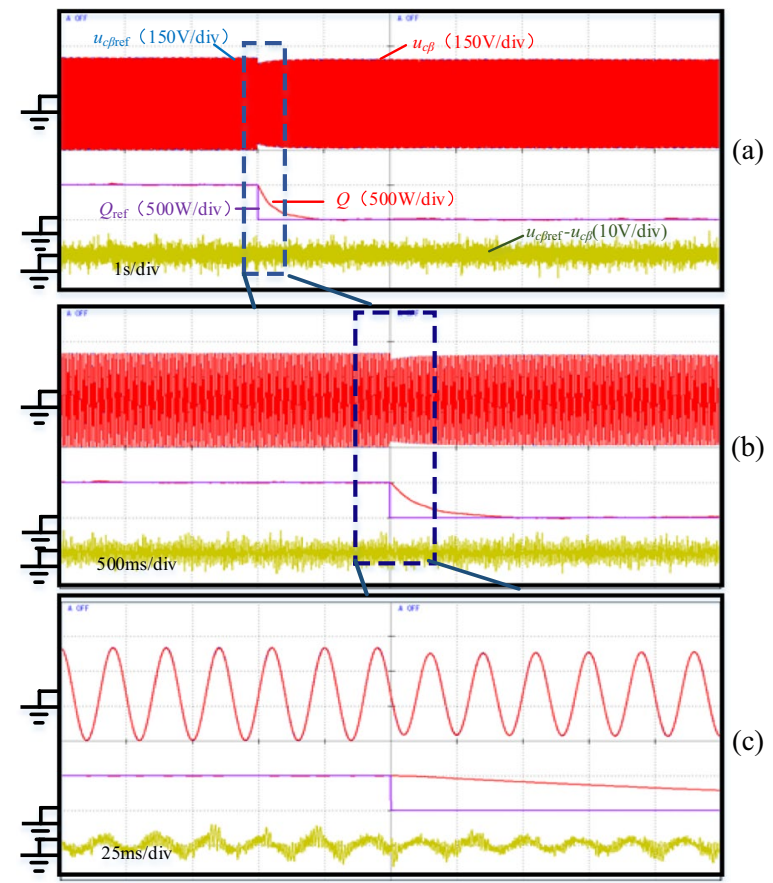

Fig. 12 Experimental waveforms during sudden change of reactive power with error compensation. a 1 s/div. b 500 ms/div. c 25 ms/div

In summary, the power outer loop with the proposed compensation algorithm can achieve the same control effect as the conventional power outer loop without compensation.

\subsection{Comparison with inaccurate parameters}

To verify that the compensation strategy can reduce the influence of model errors on system control performance, the following experiments are carried out. The reference active power $P_{\text {ref }}$ is set to $500 \mathrm{~W}$, while the reference reactive power $Q_{\text {ref }}$ is 0 Var.

Figure 13a shows the results during the sudden change of parameters. The modeled inductance and capacitance become $1 / 3$ of the actual values at time $t_{1}$. As is shown, the difference between the actual and reference $\beta$-axis voltage values becomes larger after the changes of the inductance and capacitance values. Figure $13 \mathrm{~b}$ shows a comparison of the two strategies with inaccurate parameters. $M=0$ means there is no compensation for the voltage prediction value, while $M=0.7$ means there is an error compensation for the voltage prediction value. As can be seen, the difference between the actual and reference $\beta$-axis voltage values under $M=0.7$ is much smaller than the one under $M=0$.

Therefore, choosing an appropriate value of $M$ can weaken the influence of the parameter error on voltage tracking, enhance the robustness in the face of parameter changes, and improve voltage control precision.

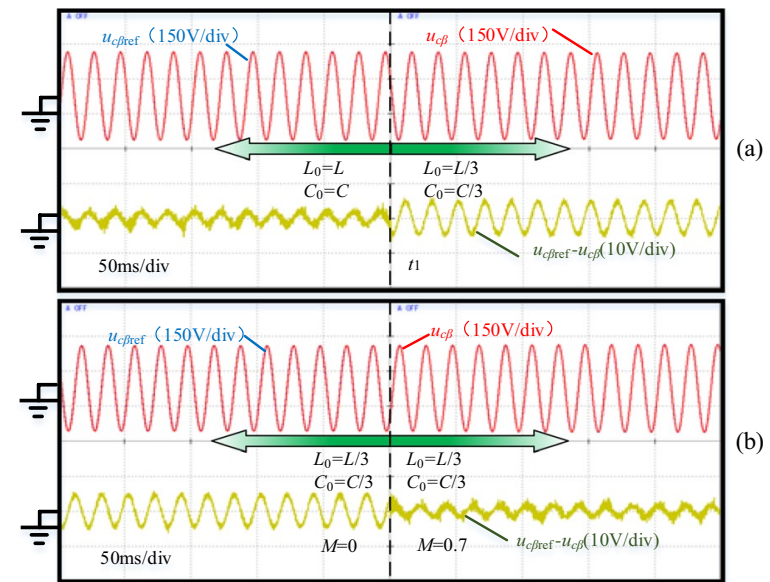

Fig. 13 a Experimental results during sudden change of parameters. b Comparison of the two strategies

With the same inductance and capacitance errors, Figs. 14 and 15 show the results during a sudden change of active power from 500 to $2000 \mathrm{~W}$, without and with error compensation, respectively. Figures $14 \mathrm{a}$ and $15 \mathrm{a}$

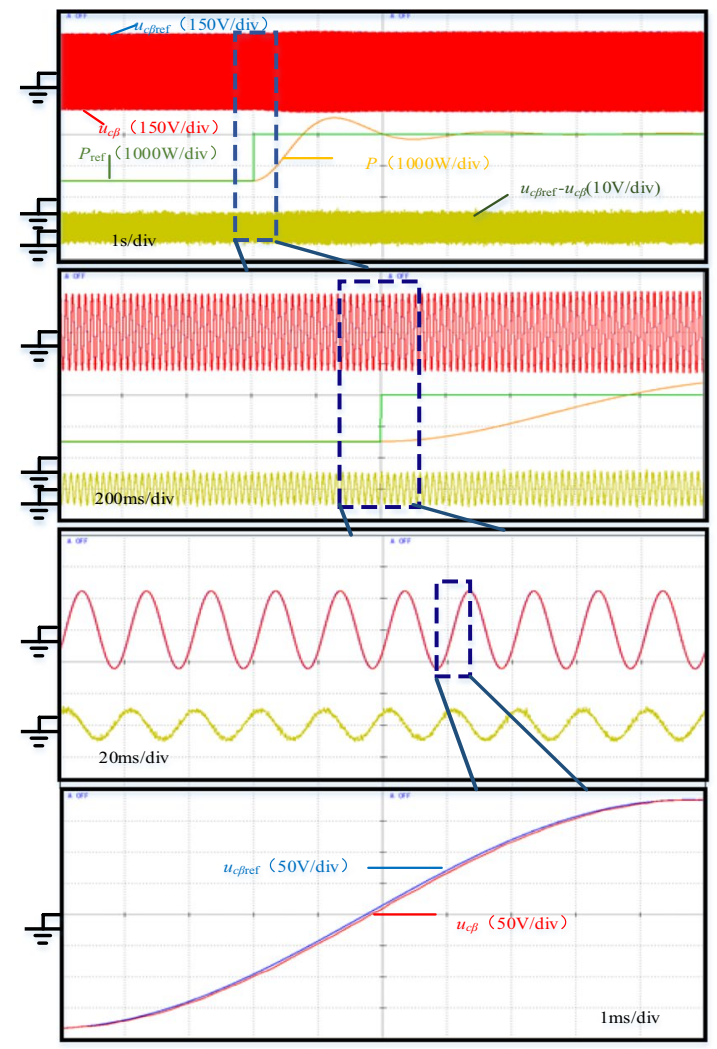

(a)

(b)

(c)

(d)

Fig. 14 Experimental waveforms during sudden change of active power without error compensation. a 1 s/div. b 200 ms/div. c 20 ms/ div. $\mathbf{d} 1 \mathrm{~ms} / \mathrm{div}$ 


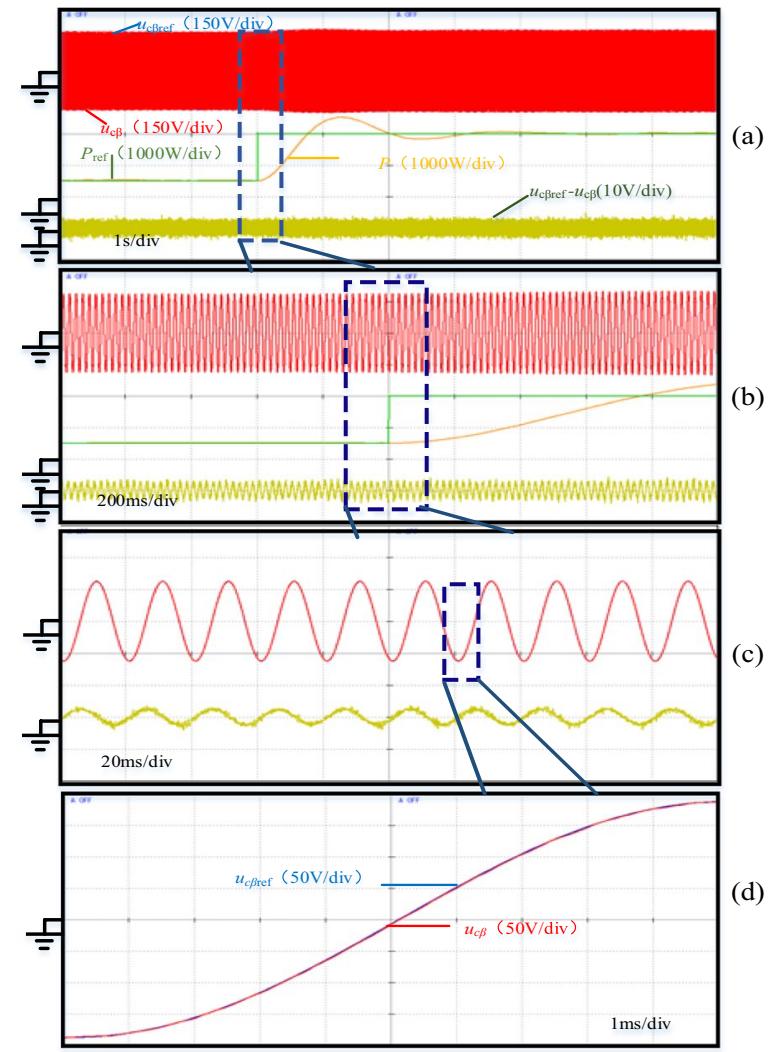

Fig. 15 Experimental waveforms during sudden change of active power with error compensation. a $1 \mathrm{~s} / \mathrm{div}$. b $200 \mathrm{~ms} / \mathrm{div}$. c $20 \mathrm{~ms} / \mathrm{div}$. d $1 \mathrm{~ms} / \mathrm{div}$

show the complete change process during the sudden change of active power, while Figs. $14 \mathrm{~b}-\mathrm{d}$ and $15 \mathrm{~b}-\mathrm{d}$ show the details of the $\beta$-axis voltage changes in different time ranges during the transients.

Comparing the steady-state differences between the actual and reference $\beta$-axis voltage before and after the sudden active power change in Figs. 14a and 15a, it can be seen that the compensation strategy can significantly reduce the voltage deviation and $\beta$-axis voltage error in steady state. In addition, by comparing Figs. 14d and 15d, it becomes apparent that the compensation strategy can minimize the voltage error caused by parameter errors, and achieve better tracking of the reference voltage in the dynamic process.

Similarly, Figs. 16 and 17 are the experimental waveforms for the two control methods when the reactive power changes from 500 to 0 Var. Again, it can be seen that the compensation strategy can significantly reduce the voltage errors caused by parameter mismatch in both steady state and in transient during a reactive power step change.

In summary, the proposed compensation strategy can reduce the influence of model errors on system control

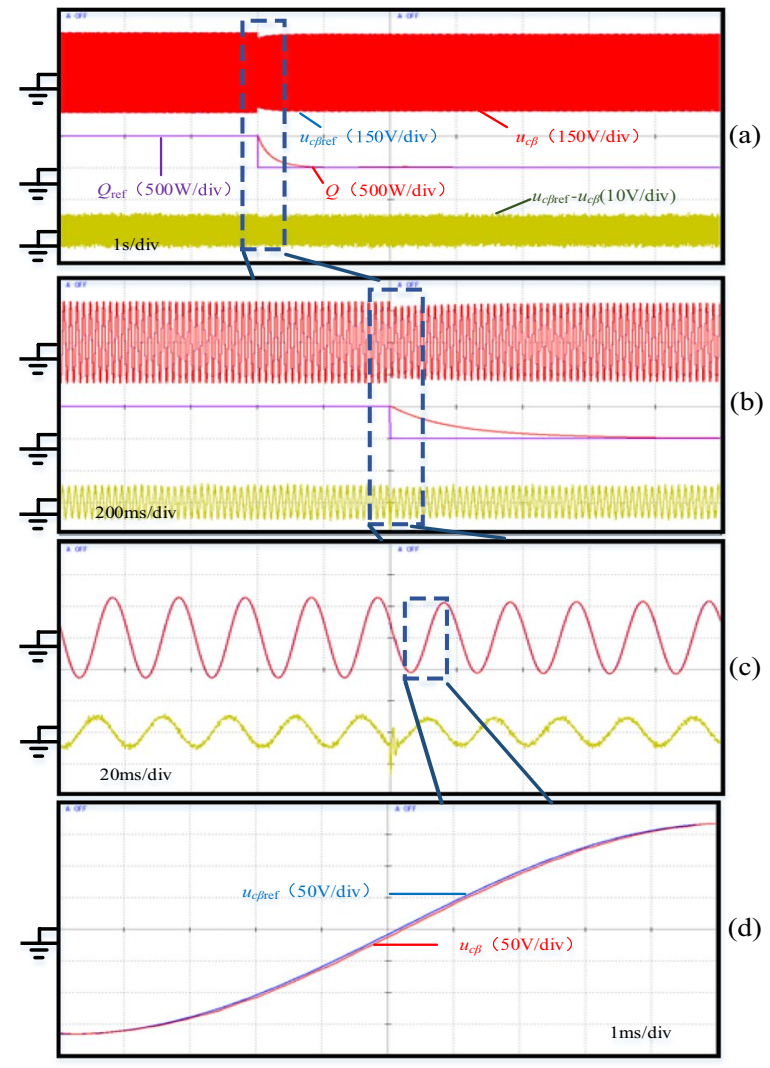

Fig. 16 Experimental waveforms during sudden change of reactive power without error compensation. a 1 s/div. b 200 ms/div. c 20 ms/ div. $\mathbf{d} 1 \mathrm{~ms} / \mathrm{div}$

performance, enhance the robustness against parameter variation in both the steady state and dynamic process.

\section{Conclusion}

A new MPC method to control the output voltage of the VSG is proposed in this paper, one with enhanced robustness against parameter variation. The important conclusions can be summarized as follows:

(1) When the parameters are perfectly matched, the proposed control strategy with compensation is almost identical to the traditional control strategy in both static and dynamic process, and both have excellent control performance.

(2) When the parameters are not matched, the predicted voltage error of the conventional MPC method will increase significantly due to its sensitivity to parameter changes.

(3) In contrast, with parameter mismatch, the proposed weighted predictive capacitor voltage control method can reduce the influence of parameter changes and improve the parameter robustness both in the steady and dynamic state. 


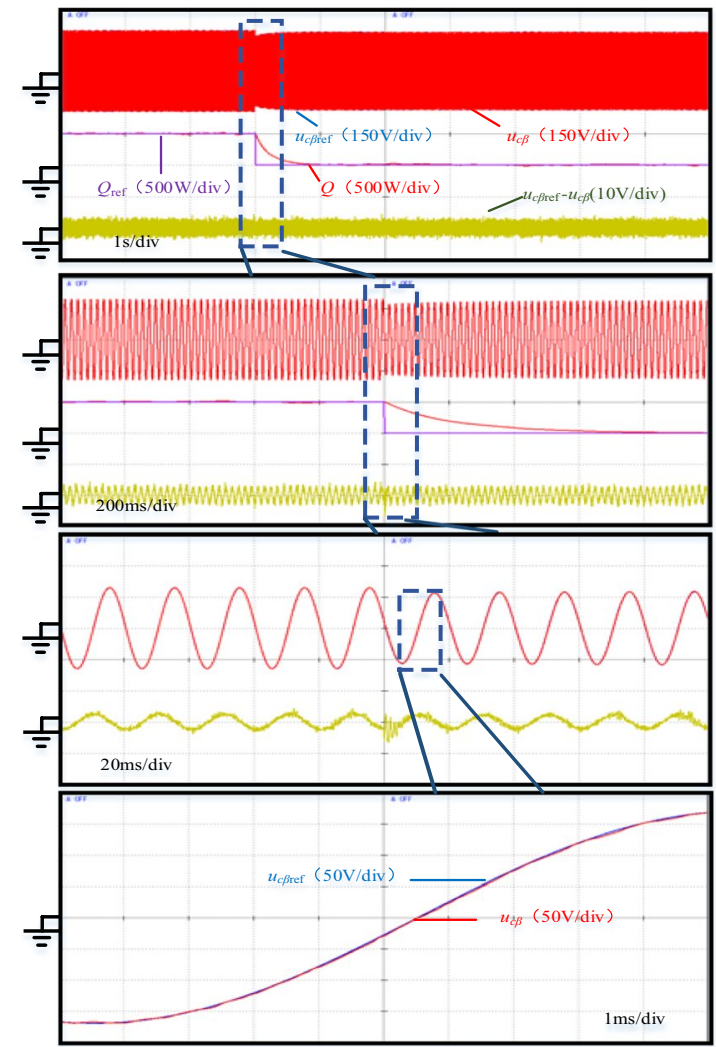

(a)

(b)

(d)

Fig. 17 Experimental waveforms during sudden change of reactive power with error compensation. a 1 s/div. b $200 \mathrm{~ms} /$ div. c $20 \mathrm{~ms} /$ div. d $1 \mathrm{~ms} / \mathrm{div}$

Finally, the effectiveness of the proposed method is verified through theoretical analysis, and its feasibility and superiority are further demonstrated through control hardware-in-the-loop experimental tests.

\section{Acknowledgements}

Not applicable.

\section{Authors' contributions}

LLG proposed the methodological framework and mathematical model, ZYX carried out theoretical analysis of the process and performed simulation and experiment to verify the proposed method. NJ, YYL and WW offered help in theory and practice, read and put forward suggestions for the paper. All authors read and approved the final manuscript.

\section{Authors' Information}

Leilei Guo received the B.S. and the Ph.D. degree in electrical engineering from the School of Electrical Engineering and Automation, Hefei University of Technology, Hefei, China, in 2010 and 2016, respectively. His current research interests include model predictive control of induction motors, permanent magnet synchronous motors, and power converters.

Zhiye $\mathbf{X u}$ is a graduate student in Zhengzhou University of Light Industry. His current research interests include model predictive control method for power converter, grid impedance identification.

Nan Jin received B.S. and M.S. degree in electrical engineering from Zhengzhou University of Light Industry, Zhengzhou, China, in 2003 and 2007, respectively, and Ph.D. degree in power electronics and electrical drives from Shanghai Jiao Tong University, Shanghai, China, in 2012. He is a professor at Zhengzhou University of Light Industry, Zhengzhou, China. His research interests include model predictive control method for power converter, fault diagnosis and tolerant control of power electronics system, wireless power transfer. Dr. Jin was the recipient of the 2018 Highlighted Paper Award from IEEE TPEL.

Yanyan Li received the Ph.D. degree in control science and engineering from the college of artificial intelligence, Tianjin key laboratory of intelligent robotics, Nankai University, Tianjin, China, in 2017. She is currently a Lecturer with Zhengzhou University of Light Industry, Zhengzhou, China. Her current research interests include model predictive control for power converters, grid impedance identification, and tolerant control of power electronics systems. Wei Wang is Major in flexible electronic materials, artificial intelligence and new energy devices. He received his Ph.D in microelectronics from Concordia University, Canada. In 2017, he joined Zhengzhou University of Light Industry, engaged in the research of artificial intelligence, tactile perception, intelligent sensor design and computer vision, and committed to the industrialization of artificial intelligence, intelligent sensor and other technologies.

\section{Funding}

This work was supported in part by the National Natural Science Foundation of China (51707176), in part by the Youth Talent Support Project of Henan Province (2019HYTP021), in part by the Youth Talent Support Project of Henan Province (2019HYTP021), in part by the Key Research, Development and Promotion Special Project (Science and Technology) of Henan Province (202102210103).

\section{Availability of data and materials}

Not applicable.

\section{Declaration}

\section{Competing interests}

The authors declare that they have no known competing financial interests or personal relationships that could have appeared to influence the work reported in this paper.

Received: 30 October 2020 Accepted: 12 November 2021

Published online: 06 December 2021

\section{References}

1. Magdy, G., et al. (2018). Microgrid dynamic security considering high penetration of renewable energy. Protection and Control of Modern Power Systems, 3, 236-246.

2. He, J. H., Liu, L., Ding, F. F., Li, C. C., \& Zhang, D. H. (2017). A new coordinated backup protection scheme for distribution network containing distributed generation. Protection and Control of Modern Power Systems, V2(1), 102-110.

3. Shahid, A. (2015). Performance evaluation of sinusoidal and space vector pulse-width-modulation for power quality enhancement in distributed generation systems. In IEEE 6th international symposium on power electronics for distributed generation systems (PEDG) (pp. 1-5).

4. Chae, W., Kim, J., Cho, J., \& Park, J. (2012). Optimal interconnection device for distributed energy resources of customer. In 3rd IEEE international symposium on power electronics for distributed generation systems (PEDG) (pp. 878-882).

5. Quintero, G. M., Reddy Challapuram, Y., Bilbao, A., Bayne, S. B., Subburaj, A. S., \& Harral, M. A. (2019). Micro-grid system modeling efforts using $\mathrm{PQ}$-control for single-phase and three-phase inverter. In IEEE international telecommunications energy conference (INTELEC) (pp. 1-5).

6. Mahesh Nair, M. P., Shetty, S. K., Raiker, G. A., Subba Reddy, B., \& Umanand, L. (2020). Three-phase five-level grid synchronized PV inverter with MPPT for micro-grid application. In IEEE international conference on power electronics, smart grid and renewable energy (PESGRE2020), Cochin, India (pp. 1-6).

7. Aijun, C., \& Xinhai, J. (2017). A stable V/F control method for permanent magnet synchronous motor drives. In IEEE transportation electrification conference and expo, Asia-Pacific (ITEC Asia-Pacific) (pp. 1-5). 
8. De Brabandere, K., Bolsens, B., vanden Keybus, J., et al. (2007). A voltage and frequency droop control method for parallel inverters. IEEE Transactions on Power Electronics, 22(4), 1107-1115.

9. Zhang, X. L., Dong, W., Yao, G. X., \& Zhang, J. J. (2018). Test method for inertia and damping of photovoltaic virtual synchronous generator based on power angle transfer function. In 2nd IEEE conference on energy internet and energy system integration (E/2) (pp. 1-5).

10. Yu, Y., \& Hu, X. (2019). Active disturbance rejection control strategy for grid-connected photovoltaic inverter based on virtual synchronous generator. IEEE Access, 7, 17328-17336.

11. Wang, S. et al. (2019). Modeling and simulation of virtual synchronous generator for photovoltaic inverter. In IEEE innovative smart grid technologies (pp. 2870-2875).

12. Shi, R., Zhang, X., Hu, C., Xu, H., Gu, J., \& Cao, W. (2018). Self-tuning virtual synchronous generator control for improving frequency stability in autonomous photovoltaic-diesel microgrids. Journal of Modern Power Systems and Clean Energy, 06(03), 482-494.

13. Zheng, X., Liu, Y., Pang, S., Liu, Z., Li, Y.\& Wang, C. (2018). Sliding mode combined VSG control to microgrid inverters. In IECON 44th annual conference of the IEEE industrial electronics society (pp. 2453-2456).

14. Jin, N., Hu, S., Gan, C., \& Ling, Z. (2018). Finite states model predictive control for fault-tolerant operation of a three-phase bidirectional AC/DC converter under unbalanced grid voltages. IEEE Transactions on Industrial Electronics, 65(1), 819-829.

15. Guo, L., Jin, N., Gan, C., \& Luo, K. (2020). Hybrid voltage vector preselection-based model predictive control for two-level voltage source inverters to reduce the common-mode voltage. IEEE Transactions on Industrial Electronics, 67(6), 4680-4691.
16. Guo, L., Jin, N., Li, Y., \& Luo, K. (2021). A Model predictive control method for grid-connected power converters without AC voltage sensors. IEEE Transactions on Industrial Electronics, 68(2), 1299-1310.

17. Guo, L., Jin, N., Gan, C., Xu, L., \& Wang, Q. (2019). an improved model predictive control strategy to reduce common-mode voltage for two-level voltage source inverters considering dead-time effects. IEEE Transactions on Industrial Electronics, 66(5), 3561-3572.

18. Zheng, C., Dragicevic, T., \& Blaabjerg, F. (2020). Model predictive control based virtual inertia emulator for an islanded AC microgrid. IEEE Transactions on Industrial Electronics, 68, 7167-7177.

19. Dragicevic, T. (2018). Model predictive control of power converters for robust and fast operation of AC microgrids. IEEE Transactions on Power Electronics, 33(07), 6304-6317.

20. Zhang, X., Wang, Y., Yu, C., \& Guo, L. (2016). Hysteresis model predictive control for high-power grid-connected inverters with output LCL filter. IEEE Transactions on Industrial Electronics, 63(01), 246-256.

21. Niu, S., Luo, Y., Fu, W., \& Zhang, X. (2020). Robust model predictive control for a three-phase PMSM motor with improved control precision. IEEE Transactions on Industrial Electronics, 68(1), 838-849.

22. Zhang, $X$., \& Cheng, Y. (2019). Robust model predictive control for PMSM drives based on current prediction error. In IEEE international symposium on predictive control of electrical drives and power electronics (PRECEDE) ( $\mathrm{pp}$. $1-5)$.

23. Jian, W., Tong, L., Dong, L. Z., \& Guo, X. D. (2019). VSG current balance control strategy under unbalanced grid voltage. In 22nd International conference on electrical machines and systems (ICEMS) (pp. 1-6).

\section{Submit your manuscript to a SpringerOpen ${ }^{\odot}$ journal and benefit from:}

- Convenient online submission

- Rigorous peer review

- Open access: articles freely available online

- High visibility within the field

- Retaining the copyright to your article

Submit your next manuscript at $\boldsymbol{\nabla}$ springeropen.com 